\title{
53. Brain Metastasis of Lung Cancer
}

\author{
Jun-ichi KaWAFUCHI \\ Neurosurgical Department, Gunma University \\ and Hiroshi Toнyамa \\ Surgical Department, Gunma University
}

The neurological and pathological features in 16 cases of brain metastasis of lung cancer are presented. The summarized results are as follows:

1. These 16 patients were 7 males and 9 females, and the age groups were all under 5 th decade except two over 6 th decade.

2. Most of these patients have complained various neurological symptoms in the initial stage prior to detection of abnormal tumor shadows on the chest roentgenogram. The average survival time from presenting symptoms to death was merely 8 months.

3. In two cases, so-called occult pulmonary carcinoma revealing evident metastatic brain tumors and negative chest roentgenorgram have been experienced.

4. The cerebral angiography is the most suitable technic of diagnosis for location and sort of tumors, showing characteristic tumor stain or vascular shifts.

5. In our series, it has been experienced that brain metastatic tumors of two cases were removed, but the pneumonectomy of original carcinomas in the second trial were impossible in consequence of mediastinal invasions.

6. The histological classifications in these 12 cases revealed adenocarcinoma in the frequency of $75 \%$, anaplastic carcinoma in only $25 \%$, and epidermoid carcinoma in none.

\section{A Case of Ectopic Pinealoma and Brain Metastasis of Malignant Melanoma}

\author{
Norihiko KomaI, Tuyoshi Kuriyama, Taisuke HayashI \\ and Yasutomo NAKANE \\ 1st Dep. of Surgery, Wakayama Medical College
}

A rare case of brain metastasis of malignant melanoma was presented. A thirty-year-old man suffering from headache and vomiting, under- 
went right carotid angiography, which revealed a tumor stain in the right frontal lobe. Removal of the tumor performed on Oct. 20, '64. Histological findings showed an ectopic pinealoma. After the operation a small black tumor which had been noted in the right leg rapidly grew up. It was removed on Nov. 21, '64. As the diagnosis was established as a malignant melanoma by histological investigation, the regional perfusion was carried out.

Four months after the operation, he again complained of headache and vomiting, and was diagnosed brain tumor in the right parietal lobe by the carotid angiography. The tumor, which could totally be removed on Apr. 27 , '65, was found to be the metastasis of malignant melanoma.

After this operation he was free from the complaints as well as liquorhypertension.

\title{
55. Experience of Postoperative Course of a Case with Brain Tumor presenting Adrenal Insufficiency and Diabetes Insipidus
}

\author{
Kenzo Matsuoka, Tohru Uozumi, Ryoichi Nozaki, \\ Shun-nosuke Minami, Muneo Kajimura, \\ Saburo SAKaKI and Hiroshi TaKeTani \\ the First Department of Surgery, Osaka Uniuersity
}

A body, aged thirteen, with a parasellar tumor were recognized to have adrenal insufficiency, hypogonadism, hypothyroidism and diabetes insipidus. After the partial resection of the tumor, symptoms of brain herniation were observed repeatedly after pitressin injections and shock that was dramatically cured by prednisolone injection were observed in dehydration stage with hypernatremia. 